\title{
陆地生态系统磷素循环及其影响因素
}

\author{
赵 琼 ${ }^{1,2}$ 曾德慧 ${ }^{1}$ * \\ （1 中国科学院沈阳应用生态研究所大青沟沙地生态实验站,沈阳 110016) \\ (2 中国科学院研究生院, 北京 100039)
}

\begin{abstract}
摘 要 磷是生命系统的重要组成成分, 其在生态系统内的迁移转化是生态系统结构和功能的决定性因素之一。 近 20 年来, 磷在陆地生态系统内的重要性受到越来越多的关注。该文总结了国内外磷循环研究的成果, 从磷的来 源、在土壤中的存在形态和固定特性、影响因素的复杂性等方面分析了磷素循环的特点; 系统阐述了磷在陆地生态 系统各库之间及其内部, 主要是植被-土壤亚系统内的迁移转化规律及影响因素。陆地生态系统磷素循环主要是 系统内部的生物化学循环, 由植物自身的遗传特性和土壤的生物、理化性质共同控制, 不同控制因素的相对重要性 因生态系统类型、时间和空间尺度而异。文章简述了磷循环研究方法的发展及存在的局限性; 另外, 分析了干旱、 半干旱地区磷循环研究的重要性和意义; 干旱区生态系统的脆弱性及其植被、土壤特性决定了其磷素循环有其自 身的特点及研究的必要性。最后指出了当前陆地生态系统磷循环研究的发展趋势。
\end{abstract}

关键词 陆地生态系统 干旱地区 磷素循环 生物有效性 影响因素

\section{PHOSPHORUS CYCLING IN TERRESTRIAL ECOSYSTEMS AND ITS CONTROLLING FACTORS}

\author{
ZHAO Qiong ${ }^{1,2}$ and ZENG De-Hui ${ }^{1}$ \\ (1 Institute of Applied Ecology, Chinese Academy of Sciences, Shenyang 110016, China) \\ (2 Graduate School of Chinese Academy of Sciences, Beijing 100039, China)
}

\begin{abstract}
Phosphorus (P) is a key element of all life and the limiting nutrient in many ecosystems of the world. P biogeochemistry partly controls ecosystem structure and function. Over the past couple of decades, the importance of $\mathrm{P}$ biogeochemistry in controlling terrestrial ecosystem processes has received increasing attention. Advances in $\mathrm{P}$ cycling in terrestrial ecosystems were reviewed in this paper. Characteristics of $\mathrm{P}$ cycling in ecosystem were attributed to factors such as the source of $\mathrm{P}$, the chemical form, traits in soils and the complexity of its controlling factors. $\mathrm{P}$ in terrestrial ecosystems is derived mainly from weathering of apatite, and biological and chemical processes, such as microbial absorption, precipitation and adsorption by $\mathrm{Fe}$ and $\mathrm{Al}$ oxides and $\mathrm{CaCO}_{3}$, easily immobilize $\mathrm{P}$ in soil. Transformations of $\mathrm{P}$ between different chemical forms in the ecosystem, especially in the soil, and its controlling factors were discussed in detail. P cycling in terrestrial ecosystems is mainly intra-ecosystem cycling which is controlled mainly by the genotype of plant and soil properties, especially $\mathrm{pH}$ and ion concentration. The relative importance of different controlling factors differs among ecosystems and across temporal and spatial scales. Also, methods for studying $\mathrm{P}$ cycling in terrestrial ecosystems were reviewed. Pot experiments, fertilization trials and sequential extraction procedures were commonly used to test for the bioavailability of different forms of soil P; however, in situ field experiments that used resin bags, litter bags, new isotope tracer methods using ${ }^{32} \mathrm{P}$ and ${ }^{33} \mathrm{P}$, and NMR spectroscopy to study $\mathrm{P}$ dynamics and the chemical structure of soil $\mathrm{P}$ were more useful for understanding $\mathrm{P}$ cycling and plant availability. Additionally, the significance of $\mathrm{P}$ cycling in arid regions was analyzed. $\mathrm{P}$ cycling in arid regions, where vegetation is sparse and soil is infertile, are very different from humid regions, and ecosystems there are more easily limited by $\mathrm{P}$. Finally, the trends and hotspots of $\mathrm{P}$ cycling in terrestrial ecosystems are summarized for different temporal and spatial scales, different study areas and contents.
\end{abstract}

Key words Terrestrial ecosystem, Arid regions, P cycling, Bioavailability, Controlling factors 
磷是生物体的重要组成成分, 是植物必需的大 量元素之一, 以广泛多样的形式参与有机体的生命 代谢过程。磷是构成许多生物大分子如 DNA、RNA、 ADP、ATP、磷酯的关键元素, 是细胞核、细胞膜的重 要组成成分, 是光合、呼吸等重要生命过程的参与 者, 在能量咜存、迁移和转化过程中起关键性的作 用。磷在植物体内含量很低, 平均约占干重的 $0.2 \%$ （潘瑞炽和董愚得, 2001), 但其重要性不亚于碳、氮。

氮成为许多生态系统的限制因子已成为共识。 但在理论上, 磷却是生物生长的首要限制因子, 并且 磷是地质时代尺度上生物生产力的限制性养分元素 (Compton et al., 2000)。由于磷的生物地球化学循 环的复杂性和研究方法的局限性, 以往养分循环的 研究主要集中在氮循环上。近 20 年来, 磷素循环在 控制陆地生态系统生态过程中的重要性已得到更多 的认识并受到越来越广泛的关注 (Walbridge，2000)。 一方面, 在一些发达国家集约化经营的农牧生态系 统中, 由于磷肥的大量施用和畜牧业污染, 土壤中磷 过量累积, 磷流失量剧增导致水体磷的富集 (Rekolainen et al., 2002); 另一方面, 在许多自然、半自然 生态系统中, 由于土壤中磷的可利用率低, 加上 19 世纪中叶以来, 氮沉降量不断增加, 土壤中碳磷比、 氮磷比失调, 土壤性质发生变化, 土壤中的磷素有效 性不足以平衡氮素有效性 (Asner et al., 2001; He et $a l$, , 2002), 使得磷成为许多陆地生态系统生物生 长和重要生态过程的限制因子( He et al., 2002; Kellogg \& Bridgham, 2003), 尤其是热带、亚热带及一 些湿地、海岸生态系统 (Ae et al., 1990; Kellogg \& Bridgham, 2003; Sundareshwar et al ., 2003) 和有机磷 含量低的干旱区生态系统 (Sanchez et al ., 1982; Lajtha \& Schlesinger, 1988; West, 1991)。据估计, 全球 $30 \%$ 以上的耕地作物生长受到磷的限制, 我国 2/3 的土壤缺磷。作为不可再生资源, 到 2050 年, 世界 上廉价的磷资源将会耗竭 (Vance et al., 2003)。因 此必须加强对陆地生态系统磷循环过程及其影响因 素的理解以实现磷资源的可持续利用。

本文总结了国内外磷循环的研究成果, 重点从 系统外循环和内循环的角度分析了陆地生态系统磷 循环的过程及其影响因素, 指出了干旱荒漠地区磷 循环研究的意义和当前陆地生态系统磷循环研究的 发展趋势, 并回顾了磷循环研究方法的发展和存在 的问题。国内有关生态系统磷循环的研究不多, 特 别是涉及到磷循环的实验生态学还重视不够。因 此, 对这一领域的研究现状和发展趋势的介绍与分
析将有利于促进和推动我国相关领域的研究。

\section{1 陆地生态系统磷素循环的过程和特点}

磷的生物地球化学循环属沉积型循环。生态系 统中磷的主要来源不是生物作用而是源于缓慢的矿 物岩石 (主要是钻磷灰石和氟磷灰石) 的风化作用。 磷在土壤中的难溶性和难移动性使得在大多数自然 生态系统内磷的流失量很低。磷在全球尺度上的周 转时间长达 $10^{7} \sim 10^{8}$ 年, 而陆地、水体有机磷的周转 时间约为 $10^{-2} \sim 10^{0}$ 年。因此, 在较短的时间尺度 上, 磷的生物地化循环主要是生态系统 (陆地、水体) 内部的生物化学循环, 系统内部的生态过程控制着 磷的动态。陆地生态系统磷素循环指磷以各种途径 输入、输出生态系统以及磷在系统内部植物-土壤之 间、各营养级生物之间、生物体内和土壤内部的迁移 转化 (图 1)。土壤中不同形态磷的转化和植物对磷 的吸收利用一直是陆地生态系统磷素循环的重点。

土壤中磷的价态 $(+5$ 价) 很稳定, 几乎无氧化 还原作用, 但有机磷和无机磷酸盐的种类多, 并且无 机磷占全磷的比例较其它养分大得多。土壤溶液中 可溶性磷酸盐易于被土壤颗粒、有机质和其它矿物 吸附、络合、形成沉淀以及土壤微生物吸收固定 (Parfitt et al ., 1975; Stark \& Jordan, 1978)。95\% $99 \%$ 的磷以难以利用的迟效状态存在, 参与生物循 环的磷仅是土壤全磷的很小部分。土壤全磷量为 $0.5 \sim 2.0 \mathrm{mg} \cdot \mathrm{g}^{-1}$ (Sanyal \& DeDatta, 1991), 通常以化 合态或吸附态的正磷酸盐的形式存在, 由于土壤中 磷的形态的复杂性, 而且各形态 P 之间可相互转化, 目前主要是按有机、无机状态及其被植物吸收的难 易程度分类。

土壤中的无机磷按其被植物吸收的难易程度分 为 3 级。最容易被植物吸收的部分为土壤溶液中的 $\mathrm{PO}_{4}^{3-} 、 \mathrm{HPO}_{4}^{2-} 、 \mathrm{H}_{2} \mathrm{PO}_{4}^{-}$, 其含量在 $0.1 \sim 10 \mu \mathrm{mol} \cdot \mathrm{L}^{-1}$ 范围内, 很少高于 $2 \mu \mathrm{mol} \cdot \mathrm{L}^{-1}$ (Frossard et al ., 2000; Hinsinger, 2001); 其次为能被酸碱盐、螯合剂等溶 解, 并与土壤溶液中的磷酸盐通过吸附与解吸附、沉 淀与溶解过程存在着缓慢的平衡的活性磷酸盐, 主 要是吸附在 $\mathrm{Fe}_{2} \mathrm{O}_{3} 、 \mathrm{Al}_{2} \mathrm{O}_{3}$ 表面、为硅酸盐类晶体所束 缚的磷酸盐以及 $\mathrm{CaHPO}_{4} 、 \mathrm{AlPO}_{4} 、 \mathrm{FePO}_{4}$ ( Mengel \& Kirby，1982; 陆文龙等，1998)等。植物难以利用的 磷酸盐通常称之为非活性磷酸盐, 仅缓慢的被释放 到土壤活性磷咜库中, 但组成还不清楚。不同种类 的无机磷对植物生长和系统磷循环的贡献不一, 例 如 $\mathrm{AlPO}_{4}$ 是胡萝卜可利用的主要磷源, 而 $\mathrm{Ca}_{3}\left(\mathrm{PO}_{4}\right)_{2}$ 


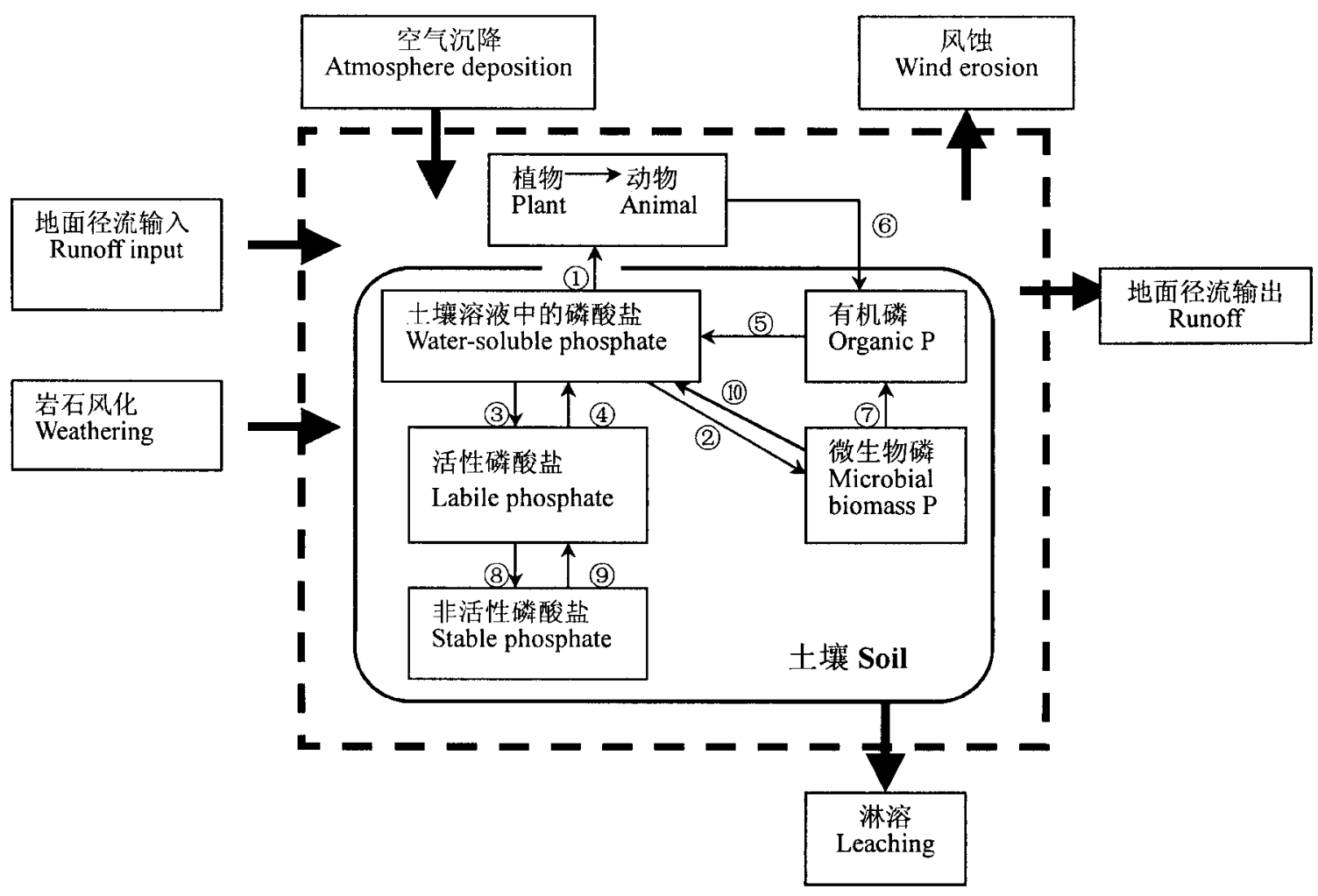

图 1 陆地生态系统磷循环图解

Fig.1 Schematic overview of phosphorus cycling in terrestrial ecosystem (Tiessen et al., 1984; Walbridge et al., 1991)

$\rightarrow$ : 系统内循环 Internal cycling $\rightarrow$ : 系统输入输出 Input and output (1): 植物吸收 Plant uptake (2): 微生物吸收 Microbial uptake (3): 吸附 和沉淀 Adsorption and precipitation (4): 解吸附、溶解 Desorption and dissolution (5): 矿化 Mineralization (6): 动植物归还 Return from biome (7)、(10): 微生物释放 Microbial release (8): 固定 Immobilization (9): 活化 Mobilization

是油菜利用的主要磷源(樊明杰等, 2001)。

土壤中的有机磷不仅有化合态的磷酸肌醇、磷 脂、核苷酸、磷蛋白、磷酸糖, 还有吸附在有机物表面 和与有机物络合的磷酸盐。土壤中有机磷约占全磷 的 20\% 90\% (Stevenson, 1986)。磷酸肌醇是最重 要的有机磷, 占有机磷总量的 $60 \%$, 另外还有近 $50 \%$ 的有机磷未鉴定出来(蒋柏藩等, 1990 )。土壤 有机磷在磷酸酶的催化下水解释放出可溶性磷酸 盐, 一部分被植物和微生物吸收, 另一部分被土壤中 的矿物固定。同时微生物通过代谢过程向土壤中释 放无机磷酸盐和低分子量的有机磷 ( He \& Zhu, 1998)。不同于有机氮的水解, 有机磷的水解仅需破 坏磷脂键, 不需要消耗太多的能量, 而且有机磷的水 解受土壤中可溶性磷酸盐的反馈调节。

土壤有效磷是生态系统磷素研究的关键, 它指 能够被植物吸收利用的那部分土壤磷, 包括土壤溶 液中的磷酸盐和能向土壤溶液中释放磷酸盐的各种 形态的有机和无机磷, 其含量由土壤内一系列的生 物和理化因素共同调节(图 1)。由于土壤中磷的形
态和反应的复杂性以及系统的自我调节作用, 有效 磷含量与土壤全磷量不一定具有相关性 (Lajtha \& Schlesinger, 1988; Kellogg \& Bridgham, 2003)。在不 同的生态系统中, 有效磷与有机磷、微生物磷和不同 形态的矿物磷的关系也不一致, 主要取决于植物可 利用的磷源。例如在我国南方的杉木 (Cunninghamia lanceolata) 人工林中, 有机磷和无机磷中的 Ca$\mathrm{P}$ 是有效磷的主要来源 (Chen, 2003)。

土壤中的磷主要以 $\mathrm{HPO}_{4}^{2-}$ 和 $\mathrm{H}_{2} \mathrm{PO}_{4}^{-}$形式被植 物吸收, 植物细胞中的磷酸根离子在 $2 \sim 20$ $\mu \mathrm{mol} \cdot \mathrm{L}^{-1}$, 显著高于土壤溶液中的浓度, 因此植物对 磷的吸收是一个主动吸收的过程 (Schachtman et $a l ., 1998$ )。磷进入植物体后大部分转化为有机物, 一小部分仍保持无机形式。磷在植物体代谢活跃的 组织 (叶肉、根尖分生组织等)和繁殖器官中含量最 多。值得注意的是, 植物地下部分与地上部分磷含 量之比可以很大, 尤其在一些草地生态系统和缺磷 的情况下。在一些草地生态系统中地下生物量磷显 著高于地上部分 (傅林谦等, 1994)。Richardson 和 
Marshall( 1996) 研究发现, 在施肥的泥炭沼泽中, 根 和地下茎中的磷占总生物量磷的 $81 \%$ 。

土壤溶液中磷酸根离子主要以扩散的方式向根 表迁移, 同时由于根系的吸收, 在根表周围易形成耗 竭圈 (Hinsinger, 2001), 因此根际与非根际土壤中全 磷和有效磷含量差异极显著 (Chen, 2003)。并且由 表层经过土壤剖面向下淋溶作用不明显, 土壤磷主 要存在于表层 $60 \mathrm{~cm}$ 范围内 (韩兴国等, 1999)。因 此, 磷较其它养分更易于成为植物生长的限制因子, 即使在全磷水平很高的情况下也不例外。

在特定的生态系统中, 不同的物种和生态过程 可能受到不同养分的限制, 在一些系统内某些植物 受氮的限制, 而另一些受磷限制 (Feller et al., 2003)。磷对生态系统的限制作用, 不仅仅限于对植 物生长的直接限制, 还可以对系统内其它组分和生 态过程起限制作用。在许多热带湿润森林和海岸系 统中, 不是植物生长, 而是微生物的生长和活动受磷 的限制, 从而使磷成为整个系统的限制因子 (Cleveland et al., 2002; Sundareshwar et al., 2003)。

\section{2 陆地生态系统磷素循环的影响因素}

2.1 生态系统整体磷素的输入-输出及其影响因素

从整个系统来看, 磷输入的途径有岩石矿物的 风化、空气沉降( 降水、气流中的土壤颗粒、花粉, 植 物、煤、石油等燃烧产生的烟雾)和地表径流输入 (图 1 )。在人工生态系统中施肥是磷素输入最主要的途 径。

岩石矿物风化是一缓慢的过程, 据估计, 通过岩 石风化进入生态系统中的磷为 $0.05 \sim 1.0$ $\mathrm{kg} \bullet \mathrm{hm}^{-2} \cdot \mathrm{a}^{-1}$, 也有报道超过 $5.0 \mathrm{~kg} \bullet \mathrm{hm}^{-2} \cdot \mathrm{a}^{-1}$ 。空 气沉降的磷酸盐以前一直被认为在系统磷循环中可 忽略, 但是一些研究发现在很多生态系统中空气沉 降是系统磷补给的一个重要来源。通过空气沉降进 入系统中的磷为 $0.07 \sim 1.7 \mathrm{~kg} \cdot \mathrm{hm}^{-2} \cdot \mathrm{a}^{-1}$ ( Newman, 1995)。因此凡是影响岩石风化与空气沉降的气候、 地形、土壤发生阶段、植被覆盖状况、物候、周围环境 条件等因素都对系统的磷输入有影响。大多数在生 态系统尺度上的磷素循环研究都采用小流域的方 法, 无需考虑地面径流输入。但对海岸生态系统和 地势低的生态系统进行研究时, 地面径流输入同输 出同等重要。

自然生态系统磷输出的途径有地表径流、沿着 土壤剖面的淋溶作用和风蚀。在存在人为干扰的生 态系统中, 植物体及其种子果实的收获是系统磷输
出的最主要途径。系统输出的磷酸盐多以颗粒物的 形式随着地表径流带出系统, 进入地表水。在森林 生态系统中随着地表径流和地下渗流输出系统的可 溶性无机磷一般不超过 $0.05 \mathrm{~kg} \cdot \mathrm{hm}^{-2} \cdot \mathrm{a}^{-1}$ ( Likens et $a l$, , 1977), 热带森林生态系统的可溶性磷输出一 般不超过 $0.3 \mathrm{~kg} \cdot \mathrm{hm}^{-2} \cdot \mathrm{a}^{-1}$ (Bruijnzeel, 1991)。但是 在农牧生态系统中, 由于磷肥、粪肥的大量施用, 土 壤磷大量流失, 并进入地表水导致富营养化。系统 流失的磷主要是正磷酸盐、磷酸双酯和磷酸单酯 (Solomon \& Lehmann, 2000)。

土壤磷流失的影响因素可归结为土壤中可溶性 磷酸盐浓度、土壤对磷的固定和缓冲能力以及外界 干扰类型和强度这几个指标。Leinwebera 等 (1999) 指出, 土壤对磷的吸附能力和饱和度预测磷的流失 比其它指标更好。当前土壤磷流失研究趋向于流失 与固定之间的阈值研究 (Pote et al., 1996; Datta, 2002)。土壤磷含量、植被覆盖状况和土壤类型 ( 岩性质)、土壤理化性质、土壤微生物状况、降雨的时 间、强度、地形对磷输出起主要作用。在不同条件下 主导因子不同, 例如在干旱荒漠地区, 植被覆盖状况 和土壤微生物是影响系统磷流失的最主要因素 ( Lajtha \& Schlesinger, 1988)。土壤类型对磷流失的影响 并没有统一的结论, 在酸性和石灰性土壤中磷流失 分别与 $\mathrm{Al}$ 和 $\mathrm{CaCO}_{3}$ 含量高度相关 (Sharpley, 1983), 而 García-Rodeja 和 Gil-Sotres (1997) 认为, Fe 含量和 土壤磷的固定特性控制着磷的流失。

在大多数自然陆地生态系统中, 磷素的输入和 输出量都很低, 并且输出量大于输入 ( Newman, 1995)。近年来, 人为活动如土地利用和管理方式的 改变(皆伐、耕作、过量施肥等) 大大增加了系统的输 入、输出量。

\section{2 生态系统内循环及其影响因素}

系统内循环主要指磷在植被-土壤亚系统内的 迁移转化过程, 由植物自身的遗传特性 (基因型) 和 土壤内的生物化学过程共同控制 (Bedford et al., 1999; Frossard et al ., 2000)。二者的作用因环境条 件、磷及其它元素的供应状况的改变而发生变化。 土壤中各种生物与化学因素的相对作用强度也因生 态系统类型 (表 1)、土壤风化程度、发育阶段、土壤 剖面层次而异。即使在同一生态系统中, 由于环境 因子的季节变化导致植物的物候变化和植被土壤特 性的季节变化, 生物与物理化学控制的相对强度也 呈现出有规律的变化。在不同的时空尺度上, 生态 系统磷素循环的控制因素也有很大差异 (Lajtha 
表 1 不同类型生态系统中土壤磷素组成和磷循环的主要影响因素

Table 1 Main forms of soil P and its controlling factors in different ecosystems

\begin{tabular}{|c|c|c|c|}
\hline $\begin{array}{l}\text { 生态系统类型 } \\
\text { Ecosystem types }\end{array}$ & $\begin{array}{l}\text { 土壤磷主要形态 } \\
\text { Main forms of soil P }\end{array}$ & $\begin{array}{c}\text { 土壤中磷素有效性的主导因子 } \\
\text { Main factors influencing P bioavailbility }\end{array}$ & $\begin{array}{l}\text { 参考文献 } \\
\text { References }\end{array}$ \\
\hline $\begin{array}{c}\text { 草地 } \\
\text { Grassland }\end{array}$ & $\begin{array}{l}\text { 无机磷占 } 70 \% \text {, 有机磷 } 27 \% \text {, 微生物磷 } 2.1 \% \\
\text { IP, OP and MP account for } 70 \%, 27 \% \text { and } 2.1 \% \text { re- } \\
\text { spectively }\end{array}$ & $\begin{array}{l}\text { 有机磷和微生物磷的转化 } \\
\text { Release from OP and MP }\end{array}$ & 傅林谦等, 1994 \\
\hline $\begin{array}{c}\text { 耕地 } \\
\text { Arable land }\end{array}$ & $\begin{array}{l}\text { 无机磷 70\%, 以 Ca-P 为主, 有机磷 30\% } \\
\text { IP (mainly Ca-P) and OP account for 70\% and 30\% }\end{array}$ & $\begin{array}{l}\mathrm{Ca}_{2}-\mathrm{P} 、 \mathrm{Al}-\mathrm{P} 、 \mathrm{Fe}-\mathrm{P} \text { 的释放 } \\
\text { Release from inorganic } \mathrm{Ca}_{2}-\mathrm{P}, \mathrm{Al}-\mathrm{P}, \mathrm{Fe}-\mathrm{P}\end{array}$ & 黄运湘等, 1994 \\
\hline $\begin{array}{c}\text { 泥炭沼泽 } \\
\text { Peatland }\end{array}$ & $\begin{array}{l}\text { 有机磷、微生物磷为主, 后者占全磷 12\% 30\% } \\
\text { Mainly MP (12\% 30\% of total P) and OP }\end{array}$ & $\begin{array}{l}\text { 调落物归还和微生物活动 } \\
\text { Decomposition of litter and release of MP }\end{array}$ & Kellogg \& Bridgham, 2003 \\
\hline $\begin{array}{l}\text { 湿润森林 } \\
\text { Humid forest }\end{array}$ & $\begin{array}{l}\text { 有机磷占 } 80 \% \text {, 无机磷中以 Al-P 为主 } \\
\text { OP } 80 \% \text { Al-P is the main IP }\end{array}$ & $\begin{array}{l}\text { 有机磷的矿化和 Ca-P 的释放 } \\
\text { Mineralization of OP and release from Ca-P }\end{array}$ & Chen, 2003 \\
\hline $\begin{array}{c}\text { 干旱荒漠 } \\
\text { Desert }\end{array}$ & $\begin{array}{l}\text { 无机磷为主, Ca-P 占全磷 50\% 以上 } \\
\text { IP dominates, Ca-P accounts for more than 50\% of to- } \\
\text { tal P }\end{array}$ & $\begin{array}{l}\text { 土壤化学过程, 主要是与 } \mathrm{CaCO}_{3} \text { 的作用 } \\
\text { Soil chemical processes relate to } \mathrm{CaCO}_{3}\end{array}$ & Lajtha \& Schlesinger, 1988 \\
\hline
\end{tabular}

IP: Inorganic P OP: Organic P MP: Microbial biomass P

\& Schlesinger, 1988; Kellogg \& Bridgham, 2003)。下 面从植物自身的遗传特性、土壤有效磷的供应能力 和植物与土壤间的相互作用 3 个主要方面来考虑系 统内磷循环的影响因素。这 3 个方面相互作用、相 互影响构成具有一定反馈和自我调节能力的整体。

\subsection{1 植物自身的遗传特性}

植物自身的遗传特性决定了植物对磷的利用效 率, 包括对土壤中磷素的活化、吸收和磷的同化利用 能力, 以及对土壤中磷含量变化的响应程度和磷素 周转率。同一植物在不同的物候期和生长阶段对磷 的需求和利用率也不同, 通常养分利用率随着年龄 的增加而增大。并且随着环境条件不断变化, 植物 对磷的利用效率有一定的调节能力。

经过长期的适应, 植物对低磷胁迫已形成两类 基本对策: 一是加强对土壤中磷的吸收能力, 增加体 内磷的含量; 二是提高自身的磷素利用效率。在低 磷胁迫时, 植物通过一系列的生理、生化、形态上的 变化来提高磷的吸收和利用效率。提高对土壤中磷 活化吸收能力的过程包括调节根系分泌物的种类和 数量、根的形态结构, 改变碳代谢 (有机酸的分泌、糖 酵解途径、线粒体呼吸、次生代谢和类囊体膜结构) 和酸性磷酸酶的合成等 (Ae et al., 1990; 㚞明杰 等, 2001); 提高同化利用能力的过程包括: 减缓生 长, 提高磷的再吸收率, 改变呼吸途径和需磷参与的 代谢途径。以上表型的变化受基因调控, 至少有 100 多个基因在植物受到低磷胁迫时同时发挥作 用, 并且这些基因共用同一调节系统 (Vance et al., 2003）。植物对磷的吸收利用效率还受到土壤中各
种形态磷含量和组成的反馈调节, 土壤中的低磷含 量是磷素利用率提高的诱导因素。

植物细胞对磷的吸收是通过跨膜的 $\mathrm{H}_{2} \mathrm{PO}_{4}^{-}$转 运蛋白实现的, $\mathrm{H}_{2} \mathrm{PO}_{4}^{-}$的吸收动力学研究表明, 植 物有两套 $\mathrm{H}_{2} \mathrm{PO}_{4}^{-}$吸收系统。 $K_{\mathrm{m}}$ 在 $50 \sim 300 \mu \mathrm{mol}$ ・ $\mathrm{L}^{-1}$ 时, 低亲和力的吸收系统起作用 (Smith et al., 2000 ); 当 $K_{\mathrm{m}}$ 在 $3 \sim 10 \mu \mathrm{mol} \cdot \mathrm{L}^{-1}$ 时, 高亲和力的吸收 系统起作用, 通过调节基因表达使转运蛋白数量增 多, 活性增强, 而且使植物光合下调, 呼吸作用下降, 生长减缓, 并诱导出糖酵解的多条旁路磷的重吸收 率也有所提高(Vance et al ., 2003)。

植物对土壤中磷的活化主要是通过改变根的形 态结构和增强与微生物的共生作用。植物的根系分 泌物 (酸性磷酸酶、有机酸、质子) 对土壤中磷的活化 起多重作用, 植物不仅能分泌磷酸酶促进有机磷的 水解, 还能分泌有机酸 (主要是一些小分子有机酸, 如柠檬酸、草酸、酒石酸)、质子以改变土壤的 $\mathrm{pH}$ 值 等理化性质来活化被固定无机磷酸盐, 如白羽扇豆 (Lupinus albus) 分泌占植物干重 $30 \%$ 的柠檬酸, 而木 豆(Cajanus cajan) 分泌番石榴酸 (Hinsinger, 2001), 而且这些小分子有机酸还能提高土壤中有效磷向根 际的扩散能力 (Gerke, 1994)。

植物生长过程中相当多的光合产物以根系分泌 物和细根脱落的形式从根中转移到土壤中, 其中很 大一部分能量用于养分的吸收。研究表明, 全球变 化导致的 $\mathrm{CO}_{2}$ 浓度的持续上升将使植物的光合作用 增强, 根系生物量增大, 植物有更大的潜力为养分利 用提供更多的能量, 从而使植物的磷素利用率提高, 
但是 $\mathrm{CO}_{2}$ 对单位根生物量的特定磷吸收率没有影响 (Campbell \& Sage, 2002)。全球气候变暖也将加快 寒冷地区养分的矿化速率从而提高养分的可利用率 (Robinson et al ., 1995)。

2.2.2 土壤有效磷的供应能力

土壤对植物可利用磷的供应能力是系统磷循环 中另一决定性环节。土壤中的一系列生物、物理、化 学因素相互影响, 共同控制着土壤中有机磷的分解, 无机磷的吸附与解吸附、沉淀与溶解过程。

影响土壤有效磷供应能力的生物因素主要是根 系和土壤微生物的活动。土壤中的菌根, 主要是 VA 菌根的活动对土壤中磷的转化和对磷素的保持和缓 冲能力的维持很重要。菌根菌丝对磷酸盐的亲合力 大, 而且可大大增加植物对磷的吸收范围, 菌丝还可 以酸化菌丝际从而促进难溶性矿物磷的溶解。研究 表明, 植物与 VA 菌根的这种共生关系是由植物控 制的, 在磷酸盐含量低的土壤中, 菌根对植物生长的 影响特别明显。在缺磷的土壤中施磷肥, 植物可利 用磷含量增加, 土壤中 VA 菌根的数量下降 (Koide \& $\mathrm{Li}$, 1990)。

土壤微生物生物量磷通常占有机磷总量的 $20 \% \sim 30 \%$, 远大于微生物碳和氮所占的相应比例, 并且微生物磷的周转比微生物碳氮快得多 (Kouno et $a l$., 1994)。据估计, 通过微生物量周转的磷为 5 $40 \mathrm{~kg} \cdot \mathrm{hm}^{-2} \cdot \mathrm{a}^{-1}$ (Brookes et al., 1982), 微生物磷比 无机磷更容易被植物利用。土壤微生物一方面分泌 磷酸酶催化有机磷的水解, 还能通过代谢过程释放 部分无机和有机磷, 另一方面吸收一部分可溶性磷 酸盐维持自身的生命活动。在健康的生态系统中, 微生物的这两个过程是紧密耦合相互调节的, 对土 壤中低的磷流失量起着积极作用。值得注意的是土 壤中磷酸酶是诱导酶, 其活性不仅与微生物活动有 关, 还在很大程度上受土壤供磷状况和植物需求影 响。

影响微生物生长和活动的因素包括土壤湿度、 温度、有机质等, 其中土壤湿度和有机质的结构成分 对有效磷供应起重要作用。许多研究表明, 土壤湿 度与无机磷含量呈负相关关系。这是由于土壤湿度 与微生物活动高度正相关, 土壤干燥引起微生物死 亡, 导致了微生物生物量中的磷向土壤中释放 (Magid \& Nielsen, 1992; Chen, 2003)。土壤温度对 土壤磷的作用不显著。土壤有机质是微生物矿化的 基质, 有机磷是有效磷的重要源和库, 在有效磷含量 低的生态系统中, 死有机体的 $\mathrm{C}: \mathrm{N}$ 比和 $\mathrm{C}: \mathrm{P}$ 比对土
壤有效磷的供应起着关键性作用, 死有机体的 $\mathrm{C}: \mathrm{P}$ 比控制着微生物矿化和固定之间的平衡, 进而控制 着植物有效磷的供应。当 $\mathrm{C}: \mathrm{P}$ 比值小于 200 为净矿 化, 当比值大于 300 为净生物固持 (Gahoonia \& Nielsen, 1992)。

在一些干旱荒漠地区, 土壤中有机质含量低、微 生物生物量磷不超过全磷的 $3 \%$, 甚至难以测出有 机磷含量。在这些生态系统中, 微生物磷在磷的生 物转化中可能仍起着很重要的作用, 但是对土壤和 整个系统中磷素的迁移转化所起的作用非常小。土 壤的物理、化学过程 (吸附解吸附与沉淀、溶解) 及控 制这些过程的生物、理化因素对系统磷循环起着主 导作用 (Lajtha \& Schlesinger, 1988)。

土壤的 $\mathrm{pH}$ 值是控制土壤中磷的化学形态和含 量最重要的因子, 但是 $\mathrm{pH}$ 值的实际影响取决于土 壤基质中现有的离子和矿物的含量, 包括与磷酸根 离子竞争络合与吸附位点的阴离子和可以与磷酸根 结合的 Fe、Al、Mn 等金属离子(Hinsinger, 2001)。在 低 $\mathrm{pH}$ 值的酸性土壤中, 磷酸盐易于被土壤溶液中 大量的 $\mathrm{Fe} 、 \mathrm{Al} 、 \mathrm{Mn}$ 氧化物表面吸附或为土壤中游离 的铝离子所沉淀。在高 $\mathrm{pH}$ 值的碱性或石灰性土壤 中, 通常交换性 $\mathrm{Ca}^{2+}$ 和 $\mathrm{CaCO}_{3}$ 含量较高, 磷酸根与 $\mathrm{Ca}^{2+}$ 形成沉淀。在中性土壤中, 土壤溶液中的磷酸 盐易于被 $\mathrm{Fe} 、 \mathrm{Al} 、 \mathrm{Mn}$ 的水化氧化物吸附。磷酸盐仅 在 $\mathrm{pH}=6.5$ 附近有效性最大。因此不同类型土壤 中可溶性磷与 $\mathrm{pH}$ 变化的关系不同。在 $\mathrm{pH}=4 \sim 6.5$ 范围内, 可溶性磷含量与 $\mathrm{pH}$ 值正相关, $\mathrm{pH}=6.5 \sim$ 8.3 , 可溶性磷含量与 $\mathrm{pH}$ 值负相关 (Parfitt, 1978)。 土壤 $\mathrm{pH}$ 值受根系和土壤微生物分泌的质子、有机 酸和调落物性质的调节, 根表的 $\mathrm{pH}$ 与土体相差大 到一个 $\mathrm{pH}$ 单位 (Nye \& Tinker, 1977)。其它养分的 供应状况对土壤 $\mathrm{pH}$ 值也有一定的影响, 尤其是氮 素营养起着重要作用。 $\mathrm{NH}_{4}^{+}$养分能使根际酸化, $\mathrm{NO}_{3}^{-}$反之能提高酸性土壤中磷的有效性 (Mengel \& Kirby，1982)。

虽然土壤中的磷几乎无氧化还原作用, 土壤的 氧化还原条件对磷素的有效性有很大的影响。土壤 的还原条件 (如水淹) 通过间接改变 $\mathrm{Fe}$ 和 $\mathrm{Mn}$ 离子 的价态加强磷的释放 (Scalenghe et al ., 2002)。另外 土壤颗粒大小也通过影响磷的吸附作用来改变土壤 磷素的有效性 (Solomon \& Lehmann, 2000; Asner et al., 2001)。

土壤内磷素的迁移转化与磷素本身的浓度也有 一定关系。研究表明, 在石灰性土壤中, 当土壤中可 
溶性磷酸盐浓度低时 (如, $<1 \mu \mathrm{mol} \cdot \mathrm{L}^{-1}$ ), 吸附过程 占主导作用; 浓度高时, 沉淀反应占主导地位 (Matar et al., 1992)。

\subsection{3 植被-土壤之间的相互作用}

总的来说, 生态系统的磷素循环是由植物对磷 的需求所拉动, 由土壤有效磷的持续供应来维持的。 二者之间的衔接是通过植物与土壤之间的相互作用 来实现的,包括植物对磷的吸收、植物以各种形式将 磷归还土壤及二者之间的反馈调节。植物归还土壤 磷具体途径包括: 树干径流、渗透水、枯枝落叶、细根 脱落、根系分泌物。在森林生态系统中不仅要考虑 乔木层的归还量还应考虑林下植被的归还量, 林下 植被在群落磷归还量中占很大比例 (杨玉盛等, 2002)。

生态系统内植物与土壤之间磷素循环是相互反 馈、相互适应的。在大多数生态系统中, 由于磷循环 在系统内部的局限性, 植物归还土壤的磷是有效磷 的重要来源。在健康的系统中, 植物和土壤是紧密 联系的,共同保证了系统内磷的高效利用和低流失 量。但是在人为干扰严重的生态系统中, 地上部分 归还量很少是导致土壤有效磷缺乏的最主要因素。

生态系统内动物与磷素循环的相互作用的研究 很少, 仅见于放牧的草地生态系统中过量的牲畜活 动对磷素循环的影响 (Powell et al., 2002)。另外, Schade 等 (2003) 研究了荒漠生态系统内草食昆虫 Sabinia setosa 的种群动态与植被-土壤中磷素循环关 系, 首次将系统磷素循环与动物种群动态以及食物 链联系起来。动物同植物、微生物、土壤一样都是生 态系统不可缺少的组成成分, 土壤动物及其它动物 在土壤养分的补给、矿化迁移等过程中都起着不可 忽视的作用。同时植被-土壤系统内磷素的变化对 动物的生长和群落动态也有很重要的影响。将动物 纳入生态系统磷素循环对于完善磷素循环的研究是 必要的。

\section{3 生态系统磷素循环影响因素的时空尺度效应}

磷素循环控制因素的时间尺度效应非常明显， 也是近期磷素循环研究的重点之一。在大多数生态 系统中, 在较短的时间内, 有效磷的主要来源是土壤 有机质, 因此短期的磷素的迁移转化、咜存是由生物 (植物与微生物) 因素控制的; 而在较长时间尺度上, 母岩的类型、组成等地球化学因素起控制作用 (Asner et al ., 2001; Kellogg \& Bridgham, 2003)。岩石圈 中的磷主要存在于深海底的沉积岩中, 在变质岩和 火山沉积岩中仅占很小一部分。随着土壤的发育,
全磷含量下降, 磷的化学形态也发生变化, 钻磷含量 下降, 铁磷以及被水化的铁铝氧化物胶膜包被的蓄 闭态磷含量增加。在我国长江以南的酸性土壤中, 蓄闭态磷在 $50 \%$ 以上, 砖红壤可达 $80 \%$ (蒋柏藩等, 1990)。

Wood 等(1984)指出, 土壤中磷的迁移转化的控 制因素还表现出沿着土壤剖面的分层效应, 土壤表 层磷素的移动和流失由生物因素控制, 下层由物理 化学因素控制。但是后来的许多研究表明, 各种生 物物理化学过程在土壤表层最活跃, 并未呈现明显 的分层效应。该分层效应只有在一定的情况下才能 出现, 例如灰土或存在 $\mathrm{Fe} 、 \mathrm{Al}$ 从表层往下淋溶并在 低层沉淀的灰化土中。而且土壤的年龄、气候、地貌 等因素都能影响分层效应的程度 (Lajtha \& Schlesinger, 1988; Walbridge et al., 1991)。

\section{3 陆地生态系统磷素循环研究方法}

随着磷循环研究的量化和不断深入, 其研究方 法也在不断地改进。陆地生态系统磷循环研究方法 上的重点和难点在于植物对于磷的需求量和植物是 否受磷限制的临界养分含量的确定 (Dow \& Robert, 1982); 土壤中不同形态磷的结构组成及各自的生物 有效性; 系统的尺度上, 不同环节和组分在磷循环中 的相对作用。总的来说, 研究方法有盆栽实验、施肥 试验、土壤连续浸提技术、野外原位监测、同位素示 踪、 ${ }^{31} \mathrm{P}-\mathrm{NMR}$ 波谱分析等。

盆栽实验是用于测定植物对磷的需求量和土壤 有效磷供应水平的传统方法, 这对于一年生草本作 物可能有重要的参考价值, 但是多年生木本植物生 活周期长, 在不同年龄对磷的需求以及土壤中磷的 活化和吸收能力有很大的差异, 通过盆栽实验得出 的结论不一定适合于田间条件下。同位素“ $A$ ”和 “E”值法的盆钵实验存在同样的缺陷 (史瑞和等, 1980）。在森林生态系统中, 估计植物实际吸磷量的 常用方法是根据一定时期内的生长量乘以不同器官 内的平均磷含量。

施肥试验由于其良好的可控性, 一直用来根据 植物生长对施肥的反应来确定植物是否受某一养分 限制, 包括盆栽施肥和田间施肥。对于磷而言, 由于 磷肥在土壤中的迟效性, 磷肥施用的长期效应更重 要, 只有在较长的试验周期才能获得较真实的结果, 尤其在森林生态系统中。由于植物在不同环境条件 下的适应性变化, 植物对磷需求的量化仍然没有一 个好的方法, 而且都是静态分析, 因此建立一个简便 
易行的动态监测方法非常必要。

野外原位研究是在自然条件下, 通过一些野外 控制实验来探索生态系统生态过程变化规律。在磷 循环研究中最主要的野外原位研究有阴离子交换树 脂原位吸附和凋落物原位分解实验。前者将阴离子 交换树脂颗粒装袋或树脂膜埋在田间土壤中, 测定 一段时间内树脂的吸磷量来估计土壤的磷素有效性 （Lajtha，1988）。后者是将收集的凋落物用尼龙袋装 好放在野外, 让其在自然条件下分解, 间隔一定时间 取回分析, 用来测定森林磷凋落物分解过程中的养 分变化。野外原位研究能较真实地反映自然条件下 养分变化状况, 是当前较理想的研究方法。

土壤连续浸提技术是依据不同形态磷生物有效 性的强弱, 利用不同的化学浸提剂逐级分离, 是研究 土壤磷素最常用和有效的方法。土壤有机磷的分级 研究始于 Bowman 和 Cole(1978) 提出的较完善的有 机磷分级体系, 将有机磷分为活性、中等活性、中稳 定性和高稳定性 4 级。无机磷分级的研究更早, 自 Chang 和 Jackson (1957) 提出的无机磷分级体系以 来, 针对不同的土壤得到了不断的改进和发展。 Hedley 等(1982) 提出了一种连续浸提技术, 利用不 同的浸提剂同时分离出不同生物有效性的有机磷和 无机磷, 并量化了微生物磷的数量。后来, Tiessen 等 （1983）、Tiessen 和 Moir（1993）又对 Hedley 的方法进 行了改进。当前 Bowman 和 Cole（1978）、Hedley 等 (1982)、Tiessen 等(1983) 的方法被国内外学者广泛 应用。虽然连续浸提法用不同的化学浸提剂能区分 出土壤中不同强度植物有效性的有机和无机磷, 但 却不能真实地反映植物对不同形态磷的利用状况， 而且不能提供任何有关不同形态磷结构组成的信 息。

研究生态系统磷素较新的方法是同位素示踪法 和核磁共振 (NMR)波谱分析技术。同位素 $\left({ }^{32} \mathrm{P} 、{ }^{33} \mathrm{P}\right)$ 示踪技术最早被用来研究磷在植物个体不同器官中 的迁移转化, 由于其动态监测的功能, 后来野外施用 同位素标记的肥料来研究生态系统的不同组分在磷 循环中的相对作用 ( Richardson \& Marshall, 1996; Kellogg \& Bridgham, 2003), 并取得了许多有价值的 成果。Newman 和 Tate (1980)引进 ${ }^{31} \mathrm{P}-\mathrm{NMR}$ 波谱仪用 来测定碱溶性磷 ( NaOH-EDTA) 的结构组成以来, ${ }^{31} \mathrm{P}-$ NMR 波谱仪广泛用于生态系统中磷, 尤其是有机磷 的结构组成的研究, 但国内还未见报道。
和意义

干旱半干旱地区生活着世界上约 20 亿人口。 干旱区生态系统由于所处环境的恶劣性, 植被稀疏, 生态系统脆弱, 容易受到土地利用方式变化尤其是 放牧的影响 (Verstraete \& Schwartz, 1991)。近半个世 纪以来, 人类活动和全球气候变化导致了大范围干 旱区生态系统的退化。20 世纪 90 年代以来, 科学 家对干旱半干旱地区的许多生态学问题给予了高度 关注。但长期以来, 水分被认为是干旱半干旱地区 植物生长的主要限制因子, 养分循环过程的研究尤 其是磷的研究一直被忽视 (Zaady et al., 1996)。

植物磷素营养对于植物的抗旱性和抗寒性具有 重要意义。在磷的影响下, 细胞结构成分的水化度 及其保持胶体束缚态水的能力被提高, 从而增强了 细胞抵抗植物体内水分降低的能力。并且水分对植 物生长的限制作用部分是通过对养分的限制来实现 的 (Pathep, 1955)。磷素营养与水分胁迫相互作用 研究表明, 改善植物磷素营养状况能改善根的生长、 叶片膨压、气孔导度和根的导水率, 在一定程度上缓 解了水分胁迫 (Radin, 1984; He et al., 2002)。

干旱荒漠地区, 植被稀疏, 且通常呈斑块状分 布。稀树草原是大多数干旱区的典型植被类型。由 高等植物及藻类、蓝细菌、地衣和苔藓构成的斑块促 使养分和水分积聚分布。并且干旱区土壤表层浅 薄, 有机质含量低, 分解作用缓慢, 土壤中有机磷和 微生物作用在磷素循环中起的作用很小, 不同形态 无机磷的相互转化和释放起主导作用 (Lajtha \& Schlesinger, 1988)。

干旱区土壤的生物、物理、化学性质决定了其对 磷素的固定和缓冲能力差 (Leinwebera et al., 1999), 磷素循环与热带湿润地区有较大的差异。研究表 明, 沙土的阳离子交换能力和对磷的吸附能力低, $10 \%$ 的磷是水溶性的, 磷素易于流失。并且沙土中 有效磷的释放主要是由沉淀-溶解过程控制的 (Zhang et al.，2002), 各种形态磷的季节变化较其 它类型土壤大 $4 \sim 40$ 倍。因此, 磷素被认为是干旱 区生态系统的限制性养分因子 (Lajtha \& Schlesinger, 1988; West, 1991)。例如在亚马逊盆地, $90 \%$ 的旱 地作物生长受到磷的限制 (Sanchez et al. , 1982)。 对非洲南部和西部大面积干旱区退化稀树草原研究 表明, 过牧导致的土壤磷素流失是最关键的因素, 而 过牧对土壤的其它养分和水分的保持能力没有影 响。土壤磷流失的进一步加剧将会导致牲畜的贫营 
养( Smith，2000)。在降雨量少且降雨集中的干旱、 半干旱地区, 通过控制磷素供应状况可以在一定程 度上保证生态系统的生产力 (He et al., 2002)。

干旱地区的生物以多种多样的生存对策来应付 不良环境 (Aerts, 1990), 通过研究这些生物、生态系 统养分对策和种群营养关系对于理解生态系统养分 循环和生态系统结构功能、促进生态恢复将会起到 积极的作用。

\section{5 小结与展望}

磷素有效性的研究始终是陆地生态系统磷素循 环研究的核心, 弄清磷在系统内的迁移转化规律及 其影响因素是磷素有效性研究的关键所在。围绕着 如何提高磷素有效性以保证生态系统持续稳定的生 产力这一核心问题, 磷素循环过程和影响因素研究 的角度和尺度在不断地变化和发展, 研究趋势可归 纳为以下几个主要方面:

1)研究的不同组织水平: 从单一植物、土壤到植 物-土壤复合体的研究,再到近年来对生态系统尺度 上的系统结构、功能型、物种组成、多样性、丰富度和 演替过程等与磷素循环之间相互作用的研究以及从 根际微生态系统、细胞分子水平来探讨磷素循环的 机理和不同尺度上磷素循环耦合研究。

2)研究对象: 从土壤高度风化的热带亚热带湿 润地区、湿地海岸生态系统拓展到温带、北极、干旱、 半干旱地区生态系统。

3)研究方法: 从盆栽、施肥实验到与野外原位动 态监测相结合, 以及同位素示踪、NMR 波谱分析等 新方法的引进和探索。

4)研究内容: 从静态到时空变化; 从定性到定 量; 从单一的磷素循环研究到磷素循环与生态系统 中 $\mathrm{C} 、 \mathrm{~N}$ 及其它元素 (如 $\mathrm{Fe} 、 \mathrm{Mn} 、 \mathrm{Al} 、 \mathrm{Ca} 、 \mathrm{Zn}$ ) 循环, 水 分、温度等气候因子之间的相互作用及其对生态系 统生产力、结构功能及生态过程的耦合效应; 磷素循 环影响因素从简单相关到机理的研究; 并且随着全 球气候变化和人类活动范围和强度的扩大, 全球气 候变化、各种自然干扰和人类活动对磷素循环的影 响也是近期磷素循环研究的热点之一; 同时生态系 统内一些特定的物种 (如对系统的其它方面起的作 用很小, 磷素周转率却很高的物种和固氮植物)对系 统磷素循环的影响也引起了一些科学家的重视; 此 外以往陆地生态系统磷素循环的研究对生态系统内 动物 (包括土壤小型动物) 及其活动考虑不多, 动物 的生长和活动及动物群落在系统磷素循环中所起的
作用和对磷素循环的响应应得到更多的重视。

我国一些学者也开展了对陆地生态系统磷循环 的研究, 但深度和广度还很不够, 尤其是对森林生态 系统磷循环的研究更是缺乏。已有的研究集中在农 田和草地生态系统 (傅林谦等, 1994), 森林磷循环 的研究仅见于南方杉木人工林 (Chen, 2003) 和北方 落叶松 (Larix olgensis) 人工林( 陈立新, 2003), 而且 在方法上的探索几乎是空白。因此, 加强我国陆地 生态系统磷素循环研究, 对于维持陆地生态系统持 续稳定生产力, 解决资源、环境问题以保证社会经济 的可持续发展是十分必要的。

\section{参 考 文 献}

Ae N, Arihara J, Okada K, Yoshihara T, Johansen C (1990). Phosphorus uptake by pigeon pea and its role in cropping systems of the Indian subcontinent. Science, 248, $477 \sim 480$.

Aerts R (1990). Nutrient use efficiency in evergreen and deciduous species from heathlands. Oecologia, 84, 391 397 .

Asner GP, Townsend A, Riley WJ, Matson PA, Neff JC, Clevel CC (2001). Physical and biogeochemical controls over terrestrial ecosystem responses to nitrogen deposition. Biogeochemistry, 54, $1 \sim 39$.

Bedford BL, Walbridge MR, Aldous A (1999). Patterns in nutrient availability and plant diversity of temperate North American wetlands. Ecology, 80, 2151 2169 .

Bowman RA, Cole CV (1978). An exploratory method for fractionation of organic phosphorus from grassland soils. Soil Science, $125(2), 95 \sim 101$.

Brookes PC, Powlson DS, Jenkinson DS (1982). Measurement of microbial biomass phosphorus in soil. Soil Biology and Biochemistry, 14, 319 329 .

Bruijnzeel LA (1991) . Nutrient input-output budgets of tropical forest ecosystems, a review. Journal of Tropical Ecology, 7, 1 24.

Campbell CD, Sage RF (2002). Interactions between atmospheric $\mathrm{CO}_{2}$ concentration and phosphorus nutrition on the formation of proteoid roots in white lupin (Lupinus albus L.). Plant, Cell \& Environment, 25, $1051 \sim 1059$.

Chang SC, Jackson ML (1957). Fractionation of soil phosphorus. Soil Science, 84,133 144 .

Chen HJ (2003) . Phosphatase activity and P fractions in soils of an 18-year old Chinese fir plantation. Forest Ecology and Management, 178, $301 \sim 310$.

Chen LX (陈立新) (2003). Relationship between soil organic phosphorus forms in larch plantations and tree growth. Chinese Journal of Applied Ecology (应用生态学报)，14, 2157 2161. (in Chinese with English abstract)

Cleveland CC, Townsend AR, Schmidt SK (2002). Phosphorus limitation on microbial processes in moist tropical forests: evidence from short-term laboratory incubations and field studies. Ecosystems, 5, $680 \sim 691$.

Compton JS, Mallinson DJ, Glenn CR, Filippelli G, Follmi K, Shields G, Zanin Y (2000). Variations in the global phosphorus cycle. In: Glenn CR ed. Marine Authigenesis: From Global to 
Microbial. Society of Sedimentary Geology, Special Publication Number, 66, $21 \sim 33$.

Datta SC (2002). Threshold levels of release and fixation of phosphorus, their nature and method of determination. Communication of Soil Sciences and Plant Analysis, 33, 213 227.

Dow DL, Robert S (1982). Proposal: critical nutrient ranges from crop diagnosis. Agronomy Journal, 74, $401 \sim 403$.

Fan MS (樊明杰), Zhang FS (张福锁) (2001). The variation in plant phosphorus acquisition efficiency and its physiological mechanism. Chinese Bulletin of Life Sciences (生命科学), 13(3), $129 \sim 131$. (in Chinese with English abstract)

Feller IC, Mckee KL, Whigham DF, O'Nell JP (2003). Nitrogen vs. phosphorus limitation across an ecotonal gradient in a mangrove forest. Biogeochemistry, 62, $145 \sim 175$.

Frossard E, Condron LM, Oberson A, Sinaj S, Fardeau JC (2000). Process governing phosphorus availability in temperate soils. Journal of Environmental Quality, 29, $15 \sim 23$.

Fu LQ (傅林谦), Zu TC (祝廷成) (1994). Phosphorus budgets and cycling in Leymunes chinensis grassland. Acta Phytoecologica Sinica (植物生态学报), 18, $290 \sim 296$. (in Chinese with English abstract)

Gahoonia TS, Nielsen NE (1992). The effect of root-induced pH changes on the depletion of inorganic and organic phosphorus in the rhizosphere. Plant and Soil, 143, 185 191 .

García-Rodeja I, Gil-Sotres F (1997). Prediction of parameters describing phosphorus desorption kinetics in soils of Galicia (Northwest Spain). Journal of Environmental Quality, 26, 1363 1369 .

Gerke J (1994) . Kinetics of Soil Phosphate Desorption as Affected by Citric Acid. Z. Pflanzenernähr, Bodenkd, 157, $17 \sim 22$.

Han XG (韩兴国), Li LH (李凌浩), Huang JH (黄建辉) (1999). An Introduction to Biogeochemistry (生物地球化学概 论). Higher Education Press, Beijing, $253 \sim 254$. (in Chinese with English abstract)

He YQ, Zhu YG, Smith SE, Smith FA (2002). Interactions between soil moisture content and phosphorus supply in spring wheat plants grown in pot culture. Journal of Plant Nutrition, 25, 913 $\sim 925$.

He ZL, Zhu J (1998). Microbial utilization and transformation of phosphate adsorbed by variable charge minerals. Soil Biology and Biochemistry, 30, $917 \sim 923$.

Hedley MJ, Stewart JWB, Chauhan BS (1982). Changes in inorganic and organic soil phosphorus fractions induced by cultivation practices and by laboratory incubations. Soil Science Society of America Journal, 46, $970 \sim 976$.

Hinsinger P (2001) . Bioavailability of soil inorganic P in the rhizosphere as affected by root-induced chemical changes, a review. Plant and Soil, 237, 173 195.

Huang YX (黄运湘), Zhang YZ (张杨珠), Zou YB (邹应斌), Dai ZQ (戴卓群), Zhou Q (周清), Wang KY (王开运) (1994). A study on the fractionation of inorganic phosphorus in some paddy soils of Hunang Province. Journal of Hunan Agricultural University (湖南农业大学学报)，24(2)，90 94. (in Chinese with English abstract)

Jiang BF (蒋柏藩), Shen RF (沈仁芳) (1990). Studies on the fractionation of soil inorganic phosphorus. Advances in Soil Science (土壤学进展) , 18, 1 8. (in Chinese)
Kellogg LE, Bridgham SD (2003) . Phosphorus retention and movement across an ombrotrophic - minerotrophic peatland gradient. Biogeochemistry, 63, 299 315 .

Koide RT, Li M (1990). On host regulation of the vesicular-arbuscular mycorrhizal symbiosis. New Phytologist, 114, $59 \sim 64$.

Kouno K, Lukito HP, Ando T, Brookes PC (1994). Microbial biomass $\mathrm{P}$ dynamics in soil. Transaction of 15 th World Congress of Soil Science, 5, $85 \sim 86$.

Lajtha K, Schlesinger WH (1988). The biochemistry of phosphorus cycling and phosphorus availability along a desert soil chronosequence. Ecology, 69, $24 \sim 39$.

Lajtha K (1988) . The use of ion-exchange resin bags for measuring nutrient availability in an arid ecosystem. Plant and Soil, 105, $105 \sim 111$.

Leinwebera P, Meissnerb R, Eckhardta KU, Seeger J (1999). Management effects on forms of phosphorus in soil and leaching losses. European Journal of Soil Science, 50, $413 \sim 424$.

Likens GE, Bormann FH, Pierce RS, Eaton JS, Johnson NM (1977) . Biogeochemistry of a Forested Ecosystem. Springer-Verlag, New York.

Lu WL (陆文龙), Zhang FS (张福锁), Cao YP (曹一平) (1998). Advance in phosphorus behavior in soils. Tianjin Agricultural Sciences (天津农业科学), 4, 2 7. (in Chinese with English abstract)

Magid J, Nielsen NE (1992). Seasonal variations in organic and inorganic phosphorus fractions of temperate-climate sandy soils. Plant and Soil, 144, $155 \sim 165$.

Matar A, Torrent J, Ryan J (1992) . Soil and fertilizer phosphorus and crop responses in the dryland Mediterranean zone. Advances in Soil Science, 18, $81 \sim 146$.

Mengel K, Kirby A (1982). Principle of Plant Nutrition. Agriculture Internal Potash Institute, Bern, $407 \sim 454$.

Newman EI (1995). Phosphorus inputs to terrestrial ecosystem. Journal of Ecology, 83, $713 \sim 726$.

Newman RH, Tate KR (1980). Soil characterization by ${ }^{31} \mathrm{P}$ nuclear magnetic resonance. Communications in Soil Science and Plant Analysis, 11, $835 \sim 842$.

Nye PH, Tinker PB (1977). Solute Movement in the Soil-root System. Blackwell Scientific Publications, Oxford.

Pan RC (潘瑞炽), Dong YD (董患得) (2001). Plant Physiolo$g y$ (植物生理学). Higher Education Press, Beijing, 30 (in Chinese)

Parfitt RL, Atkinson RJ, Smart RSC (1975). The mechanism of phosphate fixation by iron oxides. Soil Science Society of American Proceedings, 39, $837 \sim 841$.

Parfitt RL (1978) . Anion adsorption by soils and soil minerals. Advances in Agronomy, 30, $1 \sim 50$.

Pathep EN (1956). (Translated by Yang JH (杨景辉), Zhao QG (赵其国), Shi H (石华)) Plant Nutrition and Fertilization (in Russian). Science Press, Beijing, $17 \sim 18$. (in Chinese)

Pote DH, Daniel TC, Sharpley AN, Moore PA, Edwards DR, Nichols DJ (1996) . Relating extractable soil phosphorus to phosphorus losses in runoff. Soil Science Society of America Journal, $60,855 \sim 859$.

Powell JM, Jackson-Smith DB, Satter LD ( 2002 ). Phosphorus feeding and manure nutrient recycling on Wisconsin dairy farms. Nutrient Cycling in Agroecosystems, 62, 277 286. 
Radin JW (1984). Stomatal responses to water stress and abscisic acid in phosphorus deficient cotton plants. Plant Physiology, 76, $392 \sim 394$.

Rekolainen S, Salt CA, Bärlund I, Tattari S \& Culligan-Dunsmore M (2002) . Impacts of the management of radioactively contaminated land on soil and phosphorus losses in Finland and Scotland. Water, Air, and Soil Pollution, 139, $115 \sim 136$.

Richardson CJ, Marshall PE (1996). Processes controlling movement, storage and export of phosphorus in a fen peatland. Ecological Monographs, 56, 279 302 .

Robinson CH, Wookey PA, Parsons AN, Potter JA, Callaghan TV, Lee JA, Press MC, Welker JM (1995). Responses of plant litter decomposition and nitrogen materialization to simulated environmental change in a high Arctic polar semi-desert and a subarctic dwarf shrub heath. Oikos, 74, 503 512.

Sanchez PA, Bandy DE, Villachicia JH, Nicholaides JJ (1982). Amazon basin soils, management for continuous crop production. Science, 216, $821 \sim 827$.

Sanyal SK, DeDatta SK (1991). Chemistry of phosphorus transformations in soil. Advances in Soil Science, 16, $1 \sim 120$.

Scalenghe R, Edwards AC, AjmoneMarsan F, Bareris E (2002). The effect of reducing conditions on the solubility of phosphorus in a diverse range of European agricultural soils. European Journal of Soil Science, 53, 439 447 .

Schachtman DP, Reid RJ, Ayling SM (1998). Phosphorus uptake by plants, from soil to cell. Plant Physiology, 116, $447 \sim 453$. Schade JD, Kyle M, Hobbie SE, Fagan WF, Elser JJ (2003). Stoichiometric tracking of soil nutrients by a desert insect herbivore. Ecology Letters, 6, $96 \sim 102$.

Sharpley AN (1983). Effect of soil properties on the kinetics of phosphorus desorption. Soil Science Society of American Journal, $47,462 \sim 467$.

Shi RH (史瑞和), Bao XD (鲍小旦) (1980). Analysis of Soil and Agricultural Chemistry (土壤农化分析). Agricultural Press, Beijing, 72 75. (in Chinese with English abstract)

Smith FW, Rae AL, Hawkesford MJ (2000). Molecular mechanisms of phosphate and sulfate transport in plants. Biochimica et Biophysica Acta, 1465, $236 \sim 245$.

Smith RD (2000). Desertification from overgrazing in Botswana. World Conservation, 2, 11 12.

Solomon D, Lehmann J (2000) . Loss of phosphorus from soil in semi-arid northern Tanzania as a result of cropping, evidence from sequential extration and ${ }^{31} \mathrm{P}-\mathrm{NMR}$ spectroscopy. European Journal of Soil Science, 51,699 708 .

Stark NM, Jordan CF (1978). Nutrient retention by the root mat of an Amazonian rain forest. Ecology, 59, 434 437 .
Stevenson JF (1986). Cycles of Soil, Carbon, Nitrogen, Phosphorus, Sulfur, Micronutrients. John Wiley \& Sons, New York.

Sundareshwar PV, Morris JT, Koepfler EK, Fornwalt B (2003). Phosphorus limitation of coastal ecosystem processes. Science, $299,563 \sim 565$.

Tiessen H, Stewart JWB, Cole CV (1984) . Pathways of phosphorus transformations in soils of differing pedogenesis. Soil Science Society of America Journal, 48, 853 858 .

Tiessen H, Moir JO (1993) . Characterization of available P by sequential extraction. In: Carter MR ed. Soil Sampling and Methods of Analysis. Lewis Publishers, Boca Raton, Florida, 75 86.

Tiessen H, Stewart JWB, Moir JO (1983) . Changes in organic and inorganic phosphorus composition of two grassland soils and their particle size fractions during 60 - 90 years of cultivation. Journal of Soil Science, 34,815 823.

Vance CP, Uhde-Stone C, Allan DL (2003). Phosphorus acquisition and use, critical adaptations by plants for securing a nonrenewable resource. New Phytologist, 157, $423 \sim 447$.

Verstraete MM, Schwartz SA (1991). Desertification and global change. Vegetatio, 91, 3 13.

Walbridge MR, Richardson CJ, Swank WT (1991). Vertical distribution of biological and geochemical phosphorus subcycles in two southern Appalachian forest soils. Biogeochemistry, 13, $61 \sim 85$.

Walbridge R (2000). Phosphorus biogeochemistry. Ecology, 81, $1474 \sim 1475$.

West NE (1991). Nutrient cycling in soils of semiarid and arid regions. In: Skujins J ed. Semiarid Lands and Deserts: Soil Resource and Reclamation. Marcel Dekker, New York, 295 332.

Wood T, Bormann FH, Voigt GK (1984). Phosphorus cycling in a northern hardwood forest, biological and chemical control. Science, 27, $391 \sim 393$.

Yang YS (杨玉盛), Chen GS (陈光水), Xie JS (谢锦升), He ZM (何宗明), Chen YX (陈银秀), Huang RZ (黄荣珍) (2002) . Nutrient cycling of N and P by a mixed forest of Cunninghamia lanceolata and Tsoongiodendron odorum in subtropical China. Acta Phytoecologica Sinica (植物生态学报), 26, 437 480. (in Chinese with English abstract)

Zaady E, Groffman P, Shachak M (1996). Litter as a regulator of nitrogen and carbon dynamics in macrophytic patches in Negev desert soils. Soil Biology \& Biochemistry, 28, $39 \sim 46$.

Zhang MK, He ZL, Calvert DV, Stoffella PJ, Li YC, Lamb EM (2002) . Release potential of phosphorus in Florida sandy soils in relation to phosphorus fractions and adsorption capacity. Journal of Environmental Science and Health, 37, $793 \sim 809$.

责任编委: 欧阳 华 责任编辑: 姜联合 